\title{
Hematoma After a Fluoroscopically Guided Sacroiliac Joint Injection: A Case Report
}

\author{
Maria Menezes, MS'1 \\ Vwaire Orhurhu, $\mathrm{MD}^{2}$ \\ Raissa Quezado, MD² \\ Ahmed Shihab, MD²
}

\begin{abstract}
Background: Sacroiliac joint injection continues to play a role in the diagnosis and therapeutic management of patients with sacroiliac joint arthropathy, as it provides pain relief and improvement in function to those patients. It is considered a low-risk pain procedure with minimal to no adverse side effects. Although rare, serious com-plications such as infection and hematoma are a possibility, however. In anticoagulated patients, interventional pain physicians are advised to weigh the risks and benefits before discontinuing anticoagulation. The literature describes serious complications associated with stopping anticoagulation, such as myocardial infarction. Howev-er, we should also be mindful of the possibility of intramuscular hematoma formation in anticoagulated patients receiving a fluoroscopically guided sacroiliac joint injection.
\end{abstract}

Case Report: This case exposes the development of a 300-mL hematoma in the right gluteus maximus muscle after a fluoro-scopically guided sacroiliac joint injection in a patient who was taking warfarin. Consequently, she also developed neurologic symptoms such as new-onset urinary retention, weakness, and decreased sensation in her right leg.

Conclusion: Pain physicians should be mindful of the risks and benefits before deciding to hold or discontinue anticoagulation in patients undergoing fluoroscopically guided sacroiliac joint injection, as intramuscular hematomas are a possible complication.

Key words: Anticoagulation, hematoma, sacroiliac joint arthropathy, sacroiliac joint injection complications

\section{BACKGROUND}

Sacroiliac joint injection is considered a low-risk pain procedure that causes minimal adverse effects. The most common immediate adverse event is vasovagal reaction, and the most common delayed adverse event is injection-site soreness (1). Although rare, serious complications such as infection and hematoma are a possibility, however. One previous case report emphasized that serious complications such as osteomyelitis, spinal epidural abscess, meningitis, and endocarditis can happen after this procedure (2). Additionally, another case report highlighted the development of a major hematoma after a sacroiliac joint injection in a patient who was not on blood thinners but had an anatomical variation (3). This case exposes the development of a major hematoma and neurologic symptoms after a fluoroscopically guided sacroiliac joint injection in a patient who was taking warfarin.

From: 'Harvard Medical School, University of Sao Paulol, Boston, MA; ${ }^{2}$ Department of Anesthesia, Critical Care and Pain Medicine, Massachusetts General Hospital, Harvard Medical School, Boston, MA 
Anticoagulation guidelines from chronic pain societies advise health care providers to weigh the risk and benefits of anticoagulation therapy before performing low-risk procedures such as sacroiliac joint injection $(4,5)$. There is evidence supporting the practice that anticoagulation should not be discontinued prior to common interventional pain procedures. In a large observational study, 9 out of 2,296 patients who discontinued the use of anticoagulants suffered serious morbidity including 2 deaths (6). However, severe complications can also occur in patients who do not stop anticoagulation therapy, and we report such a finding in this case report.

This manuscript adheres to the Consensus-Based Clinical Case Reporting Guideline Development (CARE) guidelines outlined by Enhancing the Quality-Transparency of Health Research (EQUATOR) Network (7). The patient gave formal, informed, written consent before the article submission, and the article was written in accordance with the requirements of the Health Insurance Portability and Insurance Act (HIPAA).

\section{CASE}

A 61-year-old woman with a chief complaint of chronic multifocal pain, primarily secondary to sacroiliac joint arthropathy, presented to our clinic for routine follow-up. The patient's past medical history was significant for atrial fibrillation, stroke, coronary artery disease, diabetes mellitus, rheumatic heart disease, fibromyalgia, postlaminectomy syndrome, and sacroiliac joint arthropathy. She had no kidney impairment. Given a previous therapeutic response to a sacroiliac injection, she was rescheduled for bilateral sacroiliac joint injections. The patient reported taking $5 \mathrm{mg}$ of warfarin daily for her atrial fibrillation diagnosis. Aside from warfarin, she denied other anticoagulation medications; however, she was on $81 \mathrm{mg}$ of aspirin daily for antiplatelet effect. She was not on any other medications or supplements that could affect coagulation.

She had previously been seen at our pain clinic several times and had undergone different procedures including trigger point injections, sacroiliac joint injections, and epidural steroid injections. There was no documentation of procedure-related complications.

Before the procedure, the patient complained of lower back pain and lateral hip pain, throbbing in quality and rated as 7 out of 10 using the numeric rating scale for pain. The patient continued her usual warfarin and aspirin regimen on the day of the procedure. The patient was placed prone on the fluoroscopy table and her back was prepped and draped in the typical sterile fashion. The C-arm anteroposterior view identified the anterior and posterior sacroiliac joint line. With slight rotation of the C-arm, the medial and lateral joint lines were separated. The skin and subcutaneous tissue were anesthetized using $3 \mathrm{~mL}$ of $1 \%$ plain lidocaine with a 1.5-inch 25-gauge needle at the caudal end of the medial joint line. A 3.5-inch 22-gauge spinal needle with a small bend on the tip was slowly advanced towards the lower end of the joint line. Once the bony content was obtained, the needle was slid into the intraarticular space; $0.3 \mathrm{~mL}$ of radiocontrast dye was injected and an arthrogram was obtained. After negative aspiration, a total volume of $2.5 \mathrm{~mL}$, consisting of a mix of 1.0 $\mathrm{mL}$ of triamcinolone $40 \mathrm{mg} / \mathrm{mL}$ and $1.5 \mathrm{~mL}$ of $0.5 \%$ bupivacaine, was injected into her right intraarticular sacroiliac joint space. This was then repeated on the left side. In both sides, the procedure required only one successful attempt. The patient tolerated the procedure well. The patient denied any lower extremity weakness or numbness. The patient was able to ambulate upon discharge to home.

One day after the procedure, the patient called our clinic and was scheduled for an urgent follow-up visit in which she stated that overnight, she developed worsening, progressive pain primarily over the right gluteal region, with areas of swelling and tenderness. Additionally, she reported new onset of weakness and decreased sensation in her right leg. Lastly, she also noted new-onset urinary retention. During physical exam, the patient was noted to have a firm palpable mass in her right gluteal region. This mass was warm and tender to palpation. A computed tomography (CT) angiogram was performed and the result revealed a right gluteal lesion with a large intramuscular hematoma in the gluteus maximus muscle (Fig. 1). There were foci of contrast extravasation noted in the gluteus medius and maximus. Complete blood counts, electrolytes, and coagulation tests were obtained. No remarkable findings were noted in her laboratory values. One day after the procedure, her international normalized ratio (INR) was 2.0 and platelets were $263 \mathrm{~K} / \mathrm{uL}$.

The patient underwent a right gluteal hematoma evacuation. She was brought into the operating room and placed supine on the operative table. Anesthesia was induced and the patient was intubated without incident. She was then placed in the left lateral decubitus position. The patient was prepped and draped in typical sterile fashion. A Kocher-Langenbeck approach to the posterior right hip was used and the incision was carried to the 
fascia. The fascia was incised and the gluteus maximus spread with a Schnidt in the direction of its fibers. After the gluteus maximus was surgically exposed, a $300-\mathrm{mL}$ hematoma was encountered and evacuated. The muscle appeared viable with no evidence of retained hematoma. Hemostasis was achieved and the wound was packed sterilely. Two days after the procedure, her right gluteal wound was successfully closed.

Four months after the surgery, the patient visited our outpatient clinic. Aside from some difficulty with wound healing secondary to her multiple comorbidities, she recovered properly from the surgical intervention. During this clinic visit, the pain in her lower back and buttocks was very similar to her bilateral sacroiliac joint arthropathy. She received another bilateral sacroiliac joint injection with positive response. No complication was noted in this subsequent bilateral sacroiliac joint injection.

\section{Discussion}

Sacroiliac joint injections continue to play a role in the diagnosis and therapeutic management of patients with sacroiliac joint arthropathy (8). In diagnostic blocks, a local anesthetic is injected into the sacroiliac joint. If patients experience a positive analgesic response, the diagnosis of sacroiliac joint dysfunction can be established. Therapeutically, a local anesthetic is combined with corticosteroid to provide pain relief in the sacroiliac joint (9). Sacroiliac joint injection is an easy and simple procedure for which special instruments are not required; consequently, it is often performed in clinical practice.

In the case exhibited, a sacroiliac joint injection was performed in the setting of bilateral sacroiliac joint arthropathy. We hypothesize that the hematoma complication from the bilateral sacroiliac joint injection was likely secondary to warfarin use. Her neurological symptoms post procedure were most likely due to the hematoma compressing her sciatic nerve. She may also have suffered a transient nerve ischemic event that could have resulted in her right buttock tenderness, right leg weakness, and decreased sensation in her posterior right lower extremity.

Although a previously published case report suggested that anatomical variations can result in hematoma formation after a sacroiliac joint injection (3), we do not believe this was a possibility in our patient's scenario because she had previously received bilateral sacroiliac joint injection without hematoma formation. However, the experience of the previous provider may

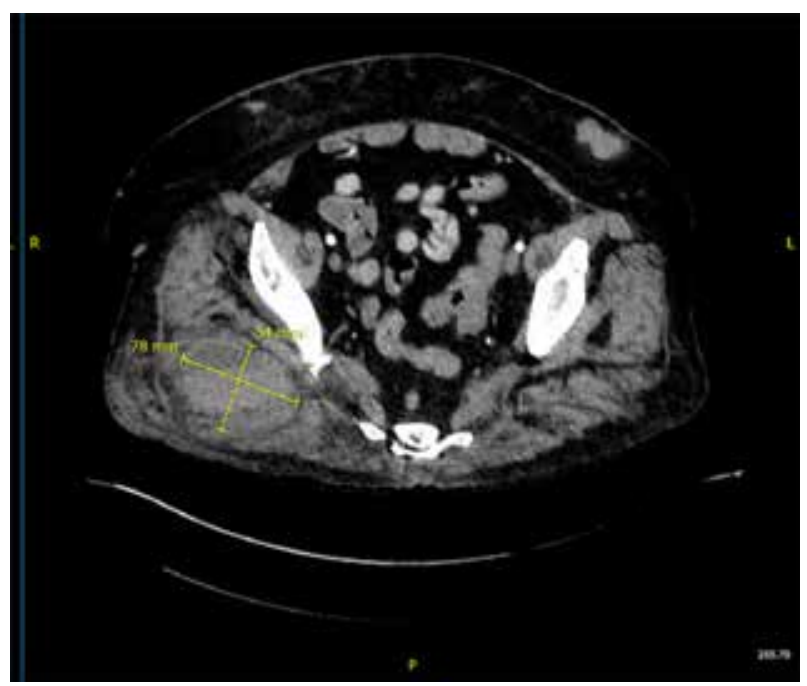

Fig. 1. Pelvic computed tomography (CT) angiogram 30 hours after sacroiliac joint injection. There is a soft tissue contusion on the right side with a large $7.8 \mathrm{~cm} \times 5.4 \mathrm{~cm}$-intramuscular hematoma in the gluteus maximus muscle.

confound this observation (3). Since the same provider performed the repetitive sacroiliac joint injection, the most likely explanation for the development of this remarkable hematoma lies in the interaction between the trauma of the procedure and a potential clotting problem induced by warfarin.

According to recommendations and guidelines from chronic pain societies, the decision to hold or continue warfarin for low-risk pain procedures is at the discretion of the treating physician(s).

There is good evidence that patients with a high risk of bleeding (e.g., old age, history of bleeding tendency, concurrent uses of other anticoagulants/antiplatelets, liver cirrhosis or advanced liver disease, and advanced renal disease) undergoing low-risk procedures should be treated as intermediate or high-risk $(4,5)$. However, there is evidence supporting the concern that discontinuation of anticoagulation before common pain procedures may not be safe. In a large observational study, 9 out of 2,296 suffered from serious morbidity, including 2 deaths (one patient had a stroke and the other had a fatal myocardial infarction) (6). In our patient, even though she had other medical conditions such as diabetes and coronary artery disease, we judged that with her past medical history of stroke and atrial fibrillation, the risk of thromboembolic events outweighed the risk of bleeding, hence our decision to keep the antithrombotic and anticoagulant regimen before the procedure. 
To date, no previous study has described a case with the development of a substantial hematoma after a low-risk pain procedure without any specific technical obstacle in a patient anticoagulated with warfarin. Occult hematomas may occur after sacroiliac joint injections. Our case highlights the need to evaluate the risk of critical hematoma formation in low-risk pain procedures such as sacroiliac joint injection. In conclusion, it is important to consider the possibility of an intramuscular hematoma after sacroiliac joint injections, especially in patients taking blood thinners, despite the fact that it is a rare complication.

\section{REFERENCES}

1. Plastaras $C T$, Joshi AB, Garvan $C$, et al. Adverse events associated with fluoroscopically guided sacroiliac joint injections. $P M R$ 2012; 4:473-478.

2. Nagpal G, Flaherty JP, Benzon HT. Diskitis, osteomyelitis, spinal epidural abscess, meningitis, and endocarditis following sacroiliac joint injection for the treatment of low-back pain in a patient on therapy for hepatitis C virus. Reg Anesth Pain Med 2017; 42:517520.

3. Adiguzel E, Kesikburun S, Yasar E, Taskaynatan MA. Major perirectal hematoma complicating sacroiliac joint injection. J Musculoskelet Pain 2014; 22:99-101.

4. Narouze S, Benzon HT, Provenzano D, et al. Interventional spine and pain procedures in patients on antiplatelet and anticoagulant medications. Reg Anesth Pain Med 2018; 43:225-262.

5. Kaye AD, Manchikanti L, Novitch MB, et al. Responsible, safe, and effective use of antithrombotics and anticoagulants in patients undergoing interventional techniques: American Society of Interventional Pain Physicians (ASIPP) Guidelines. Pain Physician 2019; 22:75-128.

6. Endres S, Shufelt A, Bogduk N. The risks of continuing or discontinuing anticoagulants for patients undergoing common interventional pain procedures. Pain Med 2017; 18:403-409.

7. Riley DS, Barber MS, Kienle GS, et al. CARE guidelines for case reports: Explanation and elaboration document. J Clin Epidemiol 2017; 89:218-235.

8. Hansen H, Manchikanti L, Simopoulos TT, et al. A systematic evaluation of the therapeutic effectiveness of sacroiliac joint interventions. Pain Physician 2012; 15:247-278.

9. Wu L, Varacallo M. Sacroiliac joint injection. In: StatPearls [Internet]. Treasure Island, FL: StatPearls Publishing; 2020. www.ncbi. nlm.nih.gov/books/NBK513245/. Date Updated 02/13/2020. 